\title{
Success in books: predicting book sales before publication
}

\author{
Xindi Wang ${ }^{1}$, Burcu Yucesoy ${ }^{1}$, Onur Varol ${ }^{1}$, Tina Eliassi-Rad ${ }^{2}$ and Albert-László Barabási ${ }^{1,2,3,4^{*}}$ (D)
}

${ }^{\text {"Correspondence: }}$
barabasi@gmail.com
${ }^{1}$ Center for Complex Network
Research and Department of
Physics, Northeastern University,
Boston, USA
${ }^{2}$ College of Computer and
Information Science, Northeastern
University, Boston, USA
Full list of author information is
available at the end of the article

available at the end of the article

\begin{abstract}
Reading remains a preferred leisure activity fueling an exceptionally competitive publishing market: among more than three million books published each year, only a tiny fraction are read widely. It is largely unpredictable, however, which book will that be, and how many copies it will sell. Here we aim to unveil the features that affect the success of books by predicting a book's sales prior to its publication. We do so by employing the Learning to Place machine learning approach, that can predicts sales for both fiction and nonfiction books as well as explaining the predictions by comparing and contrasting each book with similar ones. We analyze features contributing to the success of a book by feature importance analysis, finding that a strong driving factor of book sales across all genres is the publishing house. We also uncover differences between genres: for thrillers and mystery, the publishing history of an author (as measured by previous book sales) is highly important, while in literary fiction and religion, the author's visibility plays a more central role. These observations provide insights into the driving forces behind success within the current publishing industry, as well as how individuals choose what books to read.
\end{abstract}

Keywords: Success; Books; Learning to place

\section{Introduction}

Books, important cultural products, play a big role in our daily lives-they both educate and entertain. And it is big business: the publishing industry revenue is projected to be more than 43 billion dollars, selling more than 2.7 billion books only in the United States every year [1]. Meanwhile, authors enter a very competitive marketplace: of the over three million books published in 2015 in the United States [1], only about 4000 new titles sold more than 1000 copies within a year, and only about 500 of them became New York Times bestsellers. There are more than 45,000 published authors in the US market; while most of them struggle to get published, a few of them like J.K. Rowling earn hundreds of millions of dollars from their books [1].

The driving forces shaping the success of books have been studied by various researchers over the years, explaining the role of writing styles [2], critics [3], book reviews [4], awards [5], advertisements [6], social network [7] and word of mouth effect [8], etc. However, predicting book success from multiple factors has received much less attention. The only published study in this area focused on book sales in the German market, applying a linear model [9] and reported limited accuracy.

(c) The Author(s) 2019. This article is distributed under the terms of the Creative Commons Attribution 4.0 International License (http://creativecommons.org/licenses/by/4.0/), which permits unrestricted use, distribution, and reproduction in any medium, provided you give appropriate credit to the original author(s) and the source, provide a link to the Creative Commons license, and indicate if changes were made. 
Similar studies have focused on other cultural products, from music to movies, like using on-line reviews to forecast motion pictures sales [10], predicting the success of music and movie products by analyzing blogs [11], predicting success within the fashion industry using social media such as Instagram [12]. Nevertheless, the early-prediction of success is of great importance in cultural products. Early-prediction has been studied in various papers to address market needs for introducing new products [13], to predict movie box office success using Wikipedia [14] or to detect promoted social media campaigns [15]. Yet, predicting which cultural product will succeed before its release and understanding the mechanisms behind its success or failure remains a difficult task.

In our previous work [16], we analyzed and modeled the dynamics of book sales, identifying a series of reproducible patterns: (i) most bestsellers reach their sales peak in less than ten weeks after release; (ii) sales follow a universal "early peak, slow decay" pattern that can be described by an accurate statistical model; (iii) we showed that the formula predicted by the model helps us predict future sales. Yet, to accurately predict the future sales using the model of Ref. [16], we need at least the first 25 weeks of sales after publication, a period within which most books have already reached their peak sales and started to lose momentum. Therefore, predictions derived from this statistical model, potentially useful for long-term inventory management, are not particularly effective for foreseeing the sales potential of a new book.

In the publishing industry, limited information is available to publishers to assist their decisions on publishing (including how many copies to print, how much advance to provide, how much should they invest in marketing, etc.). Currently, publishers base their decision on the authors' previous success, the appeal of the topic, and insights from writing samples and sales of similar books, rather than relying on data specifically linked to the book considered for publication. Early-prediction of book success using the available prepublication information could be instrumental in supporting decision makers. Indeed, we would like to predict performance of a book prior to its publication. To offer such predictions, here we focus on variables available before the actual publication date, pertaining to the book's author, topic and publisher, and use machine learning to unearth their predictive power. As we show, the employed machine learning is able to accurately predict sales and to discover which features are the most influential in determining the sales of the book.

\section{Data}

Our main data source is NPD Bookscan, a data provider for the publishing industry in United States, providing meta-data including ISBN number, author name, title, category (fiction and nonfiction), Bisac code [17], publisher, price, and weekly sales of all print books published in the US since 2003. We focus on the top selling 10,000 books based on Bookscan published each month between 2008-2015 and limit our study to hardcoversthe format in which most books are published initially. We filter the data to exclude special books that are not representative of the general market (see Additional file 1). After filtering, we obtain 170,927 hardcovers published between 2008-2015. We further divide this collection into two distinct groups: baseline books published between 2008-2014 for historical sales records and statistics about genres and publishers, and evaluation books from 2015 with author's publishing history and additional information collected online for evaluating the models. Our evaluation books consist of 9702 fiction and nonfiction books published in 2015 to train and test our model. 
To supplement our model with additional online information, we collect Wikipedia pageview data for authors $[18,19]$ that counts the number of visits to each author's Wikipedia page during a given time period offering a snapshot of each author's popularity prior to the publication of their book. We also collect book descriptions created by publishing house prior to release date from Amazon and Goodreads to obtain information about a book's content that is not available from Bookscan.

\section{Machine-learning approach}

\subsection{Features}

Readers tend to choose books by authors they have read before or books written by celebrities; they often have a strong preference for specific genres and are more likely to notice well marketed books. Our features are designed and consolidated with the domain experts to capture each of these aspects of book selection.

Some of the aforementioned factors are easily quantified. For authors, visibility can be measured using Wikipedia pageviews at any given date, capturing how many people visit an author's Wikipedia page over time [14, 20-22]. The sales of an author's previous books are provided by Bookscan. The genre information is contained in the Bisac code, as discussed in Sect. 3.1.2. Topic information is produced by employing Non-negative Matrix Factorization applied to book descriptions collected from Amazon and Goodreads [23, 24]. However, it is difficult to quantify advertising. Marketing and advertising are usually the publishers' responsibility and some publishers devote more marketing resources than others. Therefore, we use the publisher as a proxy to quantify the extent of resources and know-how devoted to marketing. Publishers also play a role beyond marketing: they pass quality judgment by selecting books for publication, hence publisher quality/prestige also facilitates access to more prestigious authors and better manuscripts. Finally, we also consider seasonal fluctuations in book sales previously demonstrated as predictive [16].

In summary, we explore three feature categories: (1) author, which includes author's visibility and previous book sales; (2) book, which includes a book's genre, topic and publishing month and, (3) publisher, which captures the prominence the of book's publisher, potentially capturing its marketing and distribution power. Next, we discuss each of these feature categories separately.

\subsubsection{Author features}

Author visibility: We use Wikipedia pageviews as a proxy of the public's interest in an author, capturing his or her fame or overall visibility. There are many aspects of visibility: cumulative visibility representing all visits starting from the page's creation date is more relevant for some authors, while recent visibility is more relevant for others. To capture these multiple aspects of visibility, we explore several author-linked parameters for each book, representing the visibility feature group:

- Cumulative visibility, $F^{\text {tot }}$, counts the total pageviews of an author up until the book's publication date.

- Longevity, $t^{F}$, counts the days since the first appearance of an author's Wikipedia page until the book's publication date.

- Normalized cumulative visibility, $f^{\text {tot }}$, divides the cumulative visibility with its longevity, i.e., $f^{\text {tot }}=\frac{F^{\text {tot }}}{t^{F}}$. 
- Recent visibility, $F^{\mathrm{rec}}$, counts the total pageviews of an author during the month before the book's publication. It captures the momentary popularity of the author around publication time.

Previous sales: We use the Bookscan weekly sales data to calculate previous sales of all books written by an author. Similar to an author's visibility, we have multiple ways to incorporate previous sales. For example, previous sales in different genres from the predicted book is relevant for authors who change genres during their career (for genre information see Sect. 3.1.2). We use the following information for each book, representing the previous sales feature group:

- Total sales, $S^{\text {tot }}$, obtained by querying an author's entire publishing history from Bookscan and summing up the sales of her previous books up until the publication date of the predicted book.

- Sales in this genre, $S_{\text {in }}^{\text {tot }}$, counts the author's previous total sale in the same genre as the predicted book.

- Sales in other genres, $S_{\text {out }}^{\text {tot }}$, counts the author's previous sale in other genres.

- Career length, $t^{p}$, counts the number of days from the date of the author's first book publication till the publishing date of the upcoming book.

- Normalized sales, $s^{\text {tot }}$, normalizes the total sales based on the author's career length, i.e., $s^{\text {tot }}=\frac{S^{\text {tot }}}{t^{p}}$.

\subsubsection{Book features}

Genre information: Fiction and nonfiction books have different sales patterns as shown in previous work [16] and within fiction and nonfiction, each sub-genre may have its own behavior as well. We obtain direct information about genres from the Bisac Code [17], a standard code used to categorize books into 53 topical contents like "FICTION", "HISTORY", "COMPUTERS", etc. Under each major topic, there are more than 4000 subgenres. For example there are 189 genres under fiction, such as "FIC022000" for "FICTION / Mystery \& Detective / General". While we would like to account for each genre separately, some genres have too few books to offer representative statistics. To solve this problem, we use clustering to reduce the number of genres, aggregating genres with comparable size (i.e., number of books) and comparable potential sales. The clustering criteria is based on the number of books and the median sales of the books in each genre that are listed among top-selling (top 100) books, rather than the content of the topics. We conduct clustering on fiction and nonfiction separately using the K-means $(k=5)$ clustering algorithm [25]. Figure 1 shows the outcomes of clustering for fiction and nonfiction. For example, General Fiction and Literary are clustered to Fiction Group B. Some clusters are unexpected content-wise, like the Nonfiction Group B, which combines Religion, Business \& Economics and History. This indicates that in size and sales potential, these three genres are similar. The result of genre clustering is used to group books and calculate features.

We use various distribution descriptors (including the mean, median, standard deviations, 10th, 25th, 75th and 90th percentile, same hereafter) of book sales within each genre cluster, forming a genre cluster feature group. We form these set of features to quantify the properties of each explored distribution. 

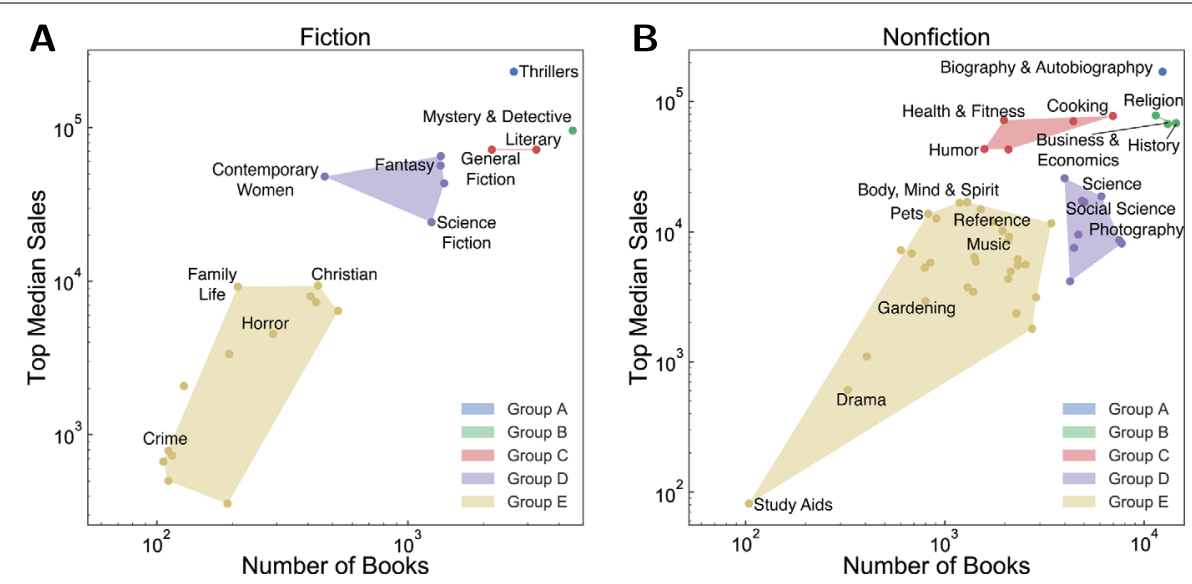

Figure 1 Clustering of genres under (A) fiction and (B) nonfiction. The results are generated with K-means algorithm with number of clusters $k=5$ for both fiction and nonfiction. The algorithm is based on the number of books and the top median sales for each genre, where the top median sales is the median sales of the top 100 most-selling books under this genre

Topic information: Genre information is assigned by publishers and can be different from how readers categorize books. For example, books under Bisac Code "BUS" (Business) can cover very different subjects, varying from finance to science of success. Therefore, we extract each book's topics from one-paragraph book summaries created by publishers, offering a better sense of the actual content of the book. We utilize Non-negative Matrix Factorization (NMF) techniques from Natural Language Processing [23, 24], which output two matrices: a topic-keyword matrix and a book-topic matrix. The topic-keyword matrix allows us to create a topic-keyword bipartite graph showing the composition of each topic as shown in Fig. 2. For each topic, we obtain the book sales distribution and the descriptors introduced in the previous section such as the mean, median, standard deviations, and different percentiles of the distributions. Then for each book, represented as a linear combination of several topics with weights assigned from the book-topic matrix, the features are calculated as a weighted average of each statistics of each topic.

Publishing month: Previous study of New York Times Bestsellers demonstrated that more books are sold during the holiday season in December [16]. We therefore aggregate all fiction and nonfiction hardcovers in our baseline books published between 2008-2014 by their publishing month, confirming that all book sales are influenced by the publication month (Fig. 3). To be specific, books published in October and November have higher sales within one year and books published in December, January or February have lower sales. To account for the role of the publication month, we develop a month feature group, where for each category (fiction and nonfiction) we obtain the book sales distribution for each month and include in the features the resulting distribution descriptors.

\subsubsection{Publisher features}

In Bookscan data, each book is assigned to a publisher and an imprint. In the publishing industry, a publishing house usually has multiple imprints with different missions. Some imprints may be dedicated to a single genre: for example Portfolio under Penguin Random House only publishes business books. Each imprint independently decides which books to 


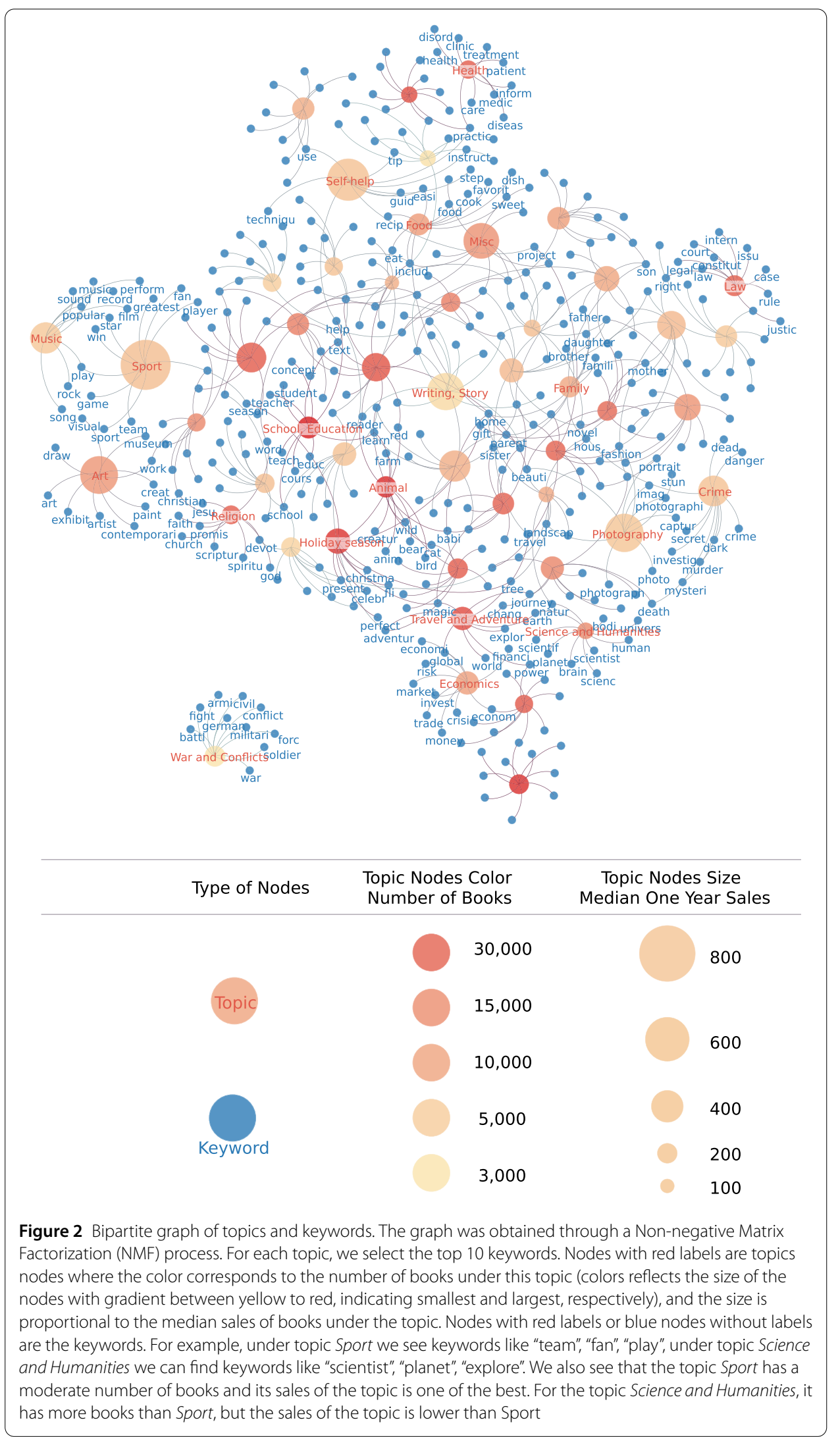


Figure 3 Seasonal fluctuations for book sales. The median of the one year sales of the top-selling books that published in the same month from 2008 to 2015. For fiction, sales increases in the summer, and October, November have the highest sales. For nonfiction, the increase in sales is not very significant over the summer months; instead October has the highest sales

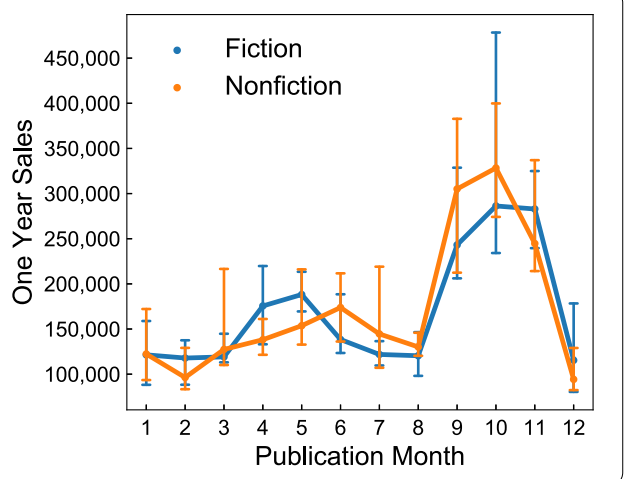

publish and takes responsibility for its editorial process and marketing. Some imprints are more attractive to authors because they offer higher advances and have more marketing resources. Additionally, more prominent imprints tend to be more selective, and books published by those imprints have higher sales.

To capture the prominence of a particular imprint, we looked at our baseline books collection published between 2008-2014, and discovered that the variation in sales within each imprint can span several orders of magnitude (Fig. 4). For example for Random House, the highest selling book sold one million copies in a year while the lowest selling book sold less than a hundred copies. Similar to publishing month, we develop an imprint feature group where for each category (fiction and nonfiction) we obtain the book sales distribution of each imprint and use the distribution descriptors as the predictive features.

\subsection{Learning algorithms}

Book sales follow a heavy-tail distribution (see Fig. 5), and in general the prediction and regression of such heavy-tailed distributions are challenging [26, 27]. Indeed, the higherorder moments and the variance of heavy-tailed distributions are not well-defined, and statistical methods based on assumptions of bounded variance leads to biased estimates. The literature on heavy-tail regression problem has developed methods based on prior correction or weighing data points [28, 29]. However, most regression methods show limited performance in learning non-linear decision boundaries and underpredict highselling books. These high selling books, however, are the most important for publishers, hence for these accuracy is the most desired.

\subsubsection{Learning to place}

To address the imbalance and heavy-tail outcome prediction problems, we employed Learning to Place algorithm [30] which addresses the following problem: Given a sequence of previously published books ranked by their sales, where would we place a new book in this sequence and estimate sales based on this placement?

Learning to Place has two stages: (1) learn a pairwise preference classifier which predicts whether a new book will sell more or less than each book in the training set; (2) given information from stage 1 , place the new book in the ordered list of previously published books sorted by their sales. Note that going from the pairwise preferences to even a partial ordering to a ranking is not trivial. The pairwise preferences may have conflicting predictions. For example, the classifier might predict that $\mathrm{A}$ is better than $\mathrm{B}, \mathrm{B}$ is better than $\mathrm{C}$, and $\mathrm{C}$ is better than A. Our majority-vote technique in the second stage is designed to resolve 


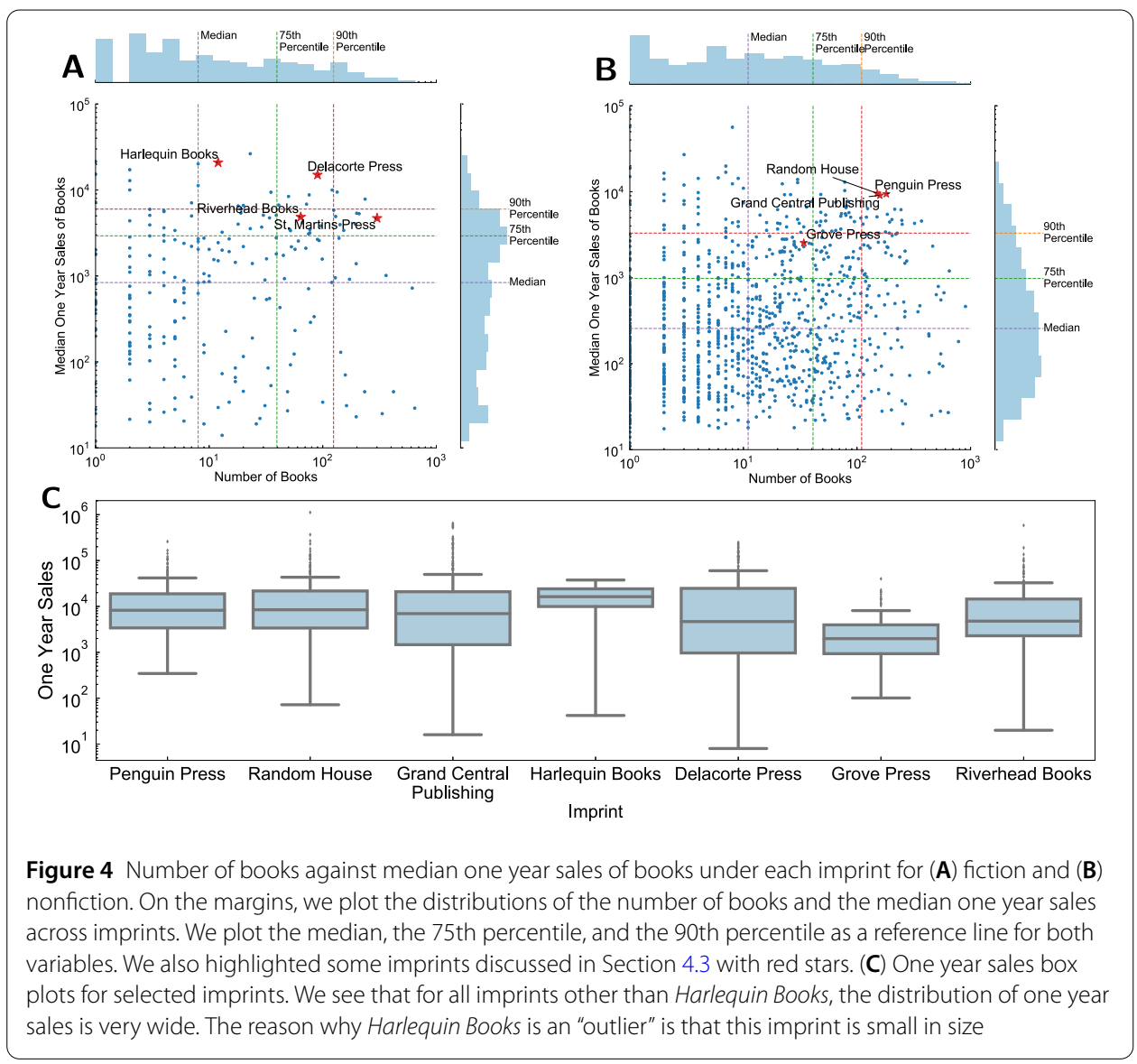

Figure 5 Sales distribution of books published between 2008-2015. The one year sales distribution is heavy-tailed, indicating that there are far more low-selling books than high selling books. Additionally, across the year, the distribution is stable

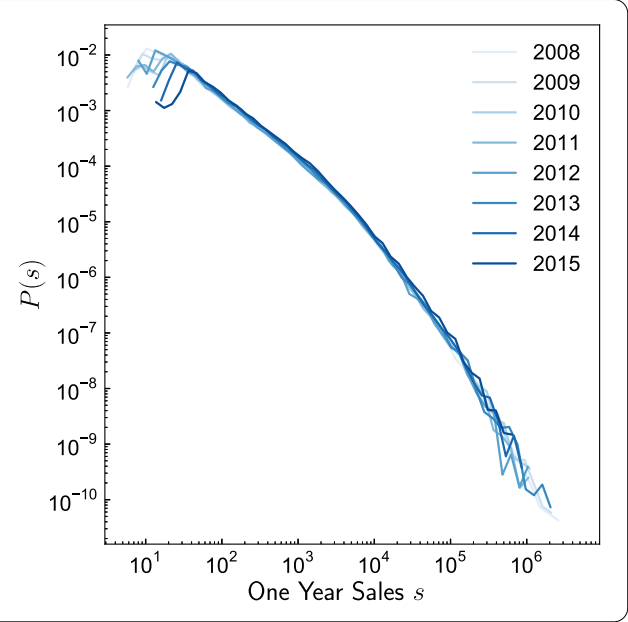

such conflicts by estimating the maximum likelihood of the data. We briefly describe two main stages of the Learning to Place algorithm and graphically explained in Fig. 6.

In the training phase, for each book pair, $i$ and $j$ with feature vectors $f_{i}$ and $f_{j}$, we concatenate the two feature vectors $X_{i j}=\left[f_{i}, f_{j}\right]$. For the target (a.k.a. response) variable, if book $i$ 's sales number is greater than book $j$ 's, we assign $y_{i j}=1$, otherwise we assign $y_{i j}=-1$ (ties are ignored in the training phase). Formally, denoting with $s_{i}$ the sales of book $i$ and with 


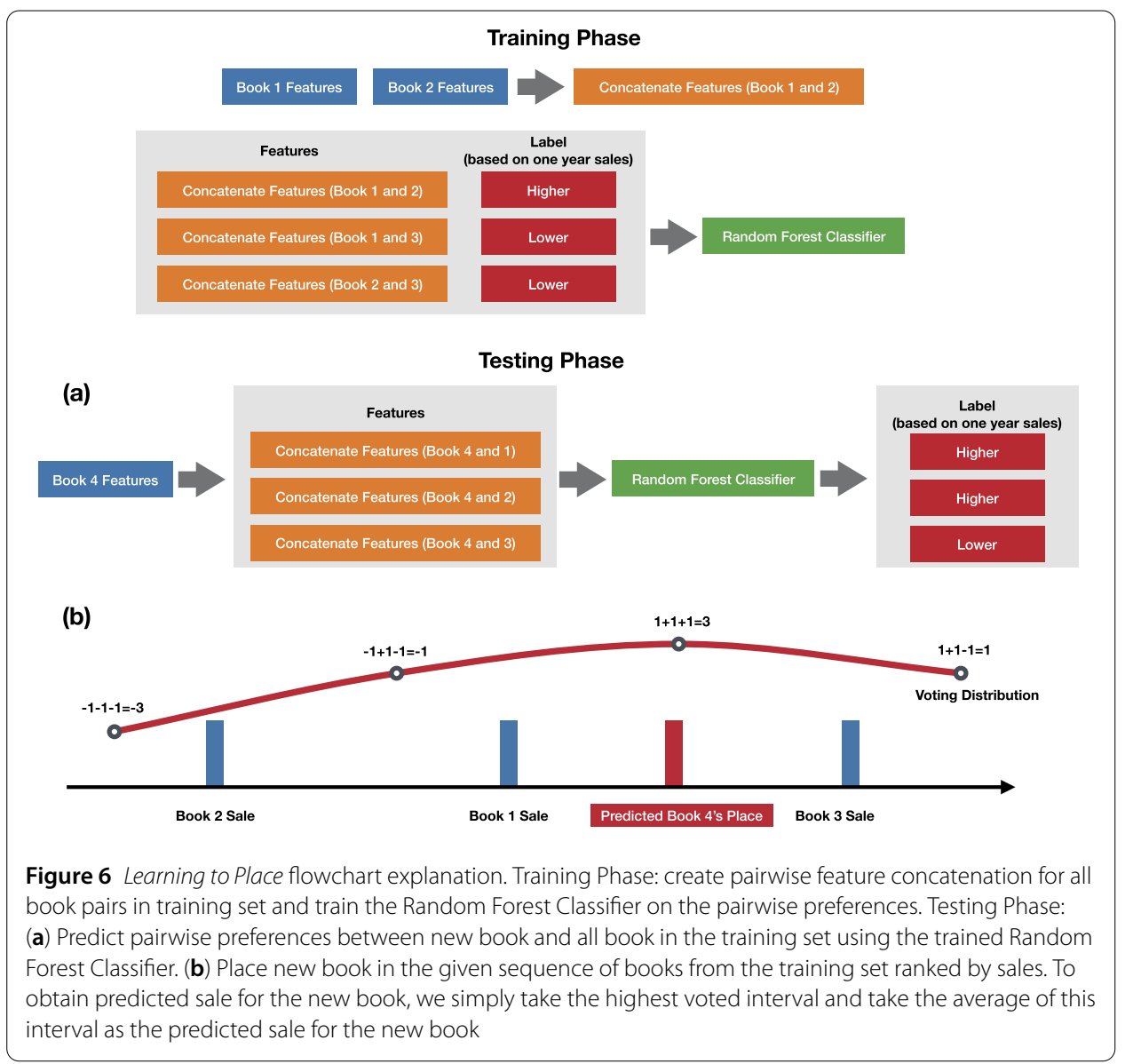

$B$ the set of books in the training set, we have the training data as:

$$
\begin{aligned}
X_{i j} & =\left[f_{i}, f_{j}\right], \quad \text { for each }(i, j) \in B \times B, i \neq j, s_{i} \neq s_{j}, \\
y_{i j} & = \begin{cases}1, & s_{i}>s_{j}, \\
-1, & s_{i}<s_{j} .\end{cases}
\end{aligned}
$$

By defining the training data, the problem is converted into a classification problem, in which we predict 1 or -1 for each book pair. Therefore we can send this training data to a classification algorithm (classifier) $F$ to fit the $y$ label (i.e., target variable) and obtain the weights on each feature in matrix $X$. In our study, we use then Random Forest Classifier [31] for this phase.

Stage 2 of Learning to Place happens during inference (i.e., testing phase). First, pairwise preferences compute using the binary classification model. For each new (test) book $k$, we obtain

$$
X_{k i}=\left[f_{k}, f_{i}\right], \quad \text { for each } i \in B \text {. }
$$


We then apply the classifier $F$ on $X_{k i}$ to obtain the predicted pairwise preference between the predicted book and all other books in the training data,

$$
\hat{y}_{k i}=F\left(X_{k i}\right) .
$$

Later Learning to Place assigns the place of the new book by treating each book in the training data as a "voter". Books (voters) from the training data are sorted by sales, dividing the sales axis into intervals. If $\hat{y}_{k i}=1$ (i.e., book $k$ should sell more than book $i$ ), sales intervals on the right of $s_{i}$ will obtain a "vote". If $\hat{y}_{k i}=-1$, book $i$ will "vote" for intervals on the left of $s_{i}$. After the voting process, we obtain a voting distribution for each test book and we take the interval with the most "votes" as the predicted sales interval for book $k$. See Fig. 6 for a depiction of the voting procedure.

\subsubsection{Baseline methods}

- Linear Regression We compare Learning to Place method with the Linear Regression method. We observe that most features we explored are heavy-tail distributed, and so are the one year sales. Therefore, we take the logarithm of our dependent and independent variables, obtaining the model:

$$
\log \left(P S_{i}\right) \sim \sum_{i} a_{i} \log \left(f_{i}\right)+\text { const }
$$

where $f_{i}$ denotes the $i$ th feature among the studied features.

- K-Nearest Neighbors (KNN) We employ regression based on $k$-nearest neighbors as an additional baseline model. The target variable is predicted by local interpolation of the targets associated with the nearest neighbors in the training set. We employed same feature transformation as in the linear regression models with an Euclidean distance metric between instances and five nearest neighbors considered $(k=5)$. The features are preprocessed in the same fashion as in Linear Regression.

- Neural Network The above two baselines do not capture nonlinear relationship between features, therefore we use a simple Multilayer Perceptron with one layer of 100 neurons as another baseline. The features are preprocessed in the same fashion as Linear Regression.

\subsection{Model testing}

To test the model, we use $k$-fold cross validation [32, 33]. We apply an evaluation method for each fold of the test sample. In our testing, we use $k=5$. For evaluation methods, we choose not to use the classic $R^{2}$ score: the book sale is heavy-tailed distributed and we are more interested in the error in the log space. $R^{2}$ is not well-defined in log space because the error does not follow a Gaussian distribution, the basic assumption behind $R^{2}$. The performance measure are as follows:

- $A U C$ and ROC: Evaluate the ranking obtained through the algorithm directly with the true ranking. We consider the true value of each train instance as a threshold and we binarize any predicted value and target value depending on this threshold. Having these two binarized lists, we compute the true positive rate (TPR) and the false positive rate (FPR) for a given threshold. For various thresholds of high- and low-sale books, we compute true positive rates and false positive rates of the ROC (Receiver 
Operating Characteristic) curve and then calculate the AUC (Area Under Curve) score (see Additional file 1).

- High-end RMSE: We calculate RMSE (Root-mean-square Error) for high-selling books to measure the accuracy of the sales prediction for high-selling books in the top 20 percentile. Since book sales follow a heavy tailed distribution, we calculate the RMSE based on the log values of the predicted and the actual sales.

\section{Results}

We evaluated Learning to Place and other baseline algorithms on hardcover books published in 2015, aiming to predict the one year sales of each book. In our experiments, we train models and evaluate their performance on leave-out fraction of the 5-fold crossvalidation.

\subsection{Predictions}

Figure 7A shows the scatter plot of actual one year sales against predicted one year sales for fiction and nonfiction. If we use Linear Regression (see Fig. 7A first column), on the high end (when true sales exceed $10^{4}$ copies) the predictions are systematically below the 45-degree reference line implying that Linear Regression systematically underpredicts the real sales. Similar underpredictions happen with KNN and Neural Network in nonfiction. However, as shown in Fig. 7A last column, Learning to Place offers improved predictive power on high-selling books.

To see this more clearly, we use a Quantile-quantile plot (Fig. 7B) for fiction and nonfiction under Learning to Place and other baseline methods. We find that for fiction, Learning to Place and Neural Network provide the closest output to the ground truth (45 degree line) while for nonfiction, Learning to Place offers the closest output to the ground truth. KNN and Linear Regression, however, fail to predict high values for books at the high-end, leading to a significant deviation from the 45 degree line at high quantiles.

Figure 8A and B show the ROC curve for fiction and nonfiction, comparing Learning to Place, Linear Regression, K-nearest neighbor, Neural Network and Random Placement. We see that the curves for Learning to Place are almost always above the curves for the other methods, indicating that Learning to Place outperforms the other approaches.

Table in Fig. 8 shows the AUC score and High-end RMSE for fiction and nonfiction, comparing K-nearest neighbor, Linear Regression, Neural Network and Learning to Place. It confirms that for both fiction and nonfiction, Learning to Place always offers higher AUC score, and lower High-end RMSE, indicating that it outperforms all explored methods. For the sake of completeness, we are reporting RMSE scores of all books (Additional file 1 Table 1) and our proposed method achieves the lowest RMSE score.

\subsection{Feature importance}

Feature importance for fiction and nonfiction: To identify the relative importance of specific feature groups, we plot the AUC score using each feature group for fiction and nonfiction, shown in Fig. 9A. It is remarkable how similar the curves are, suggesting that the driving forces determining book sales are rather universal. We can also see that for both fiction and nonfiction, Imprint is the most important feature group. However, fiction relies slightly more on previous sales and visibility than nonfiction, while nonfiction relies slightly more on the imprint prestige. 

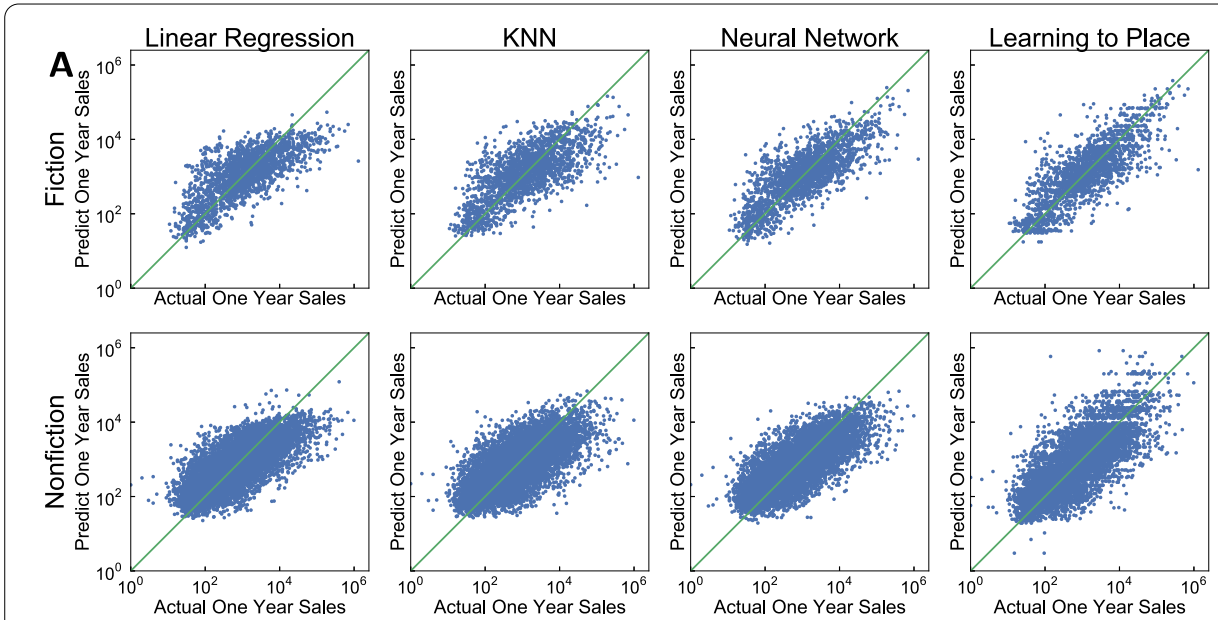

\section{B}
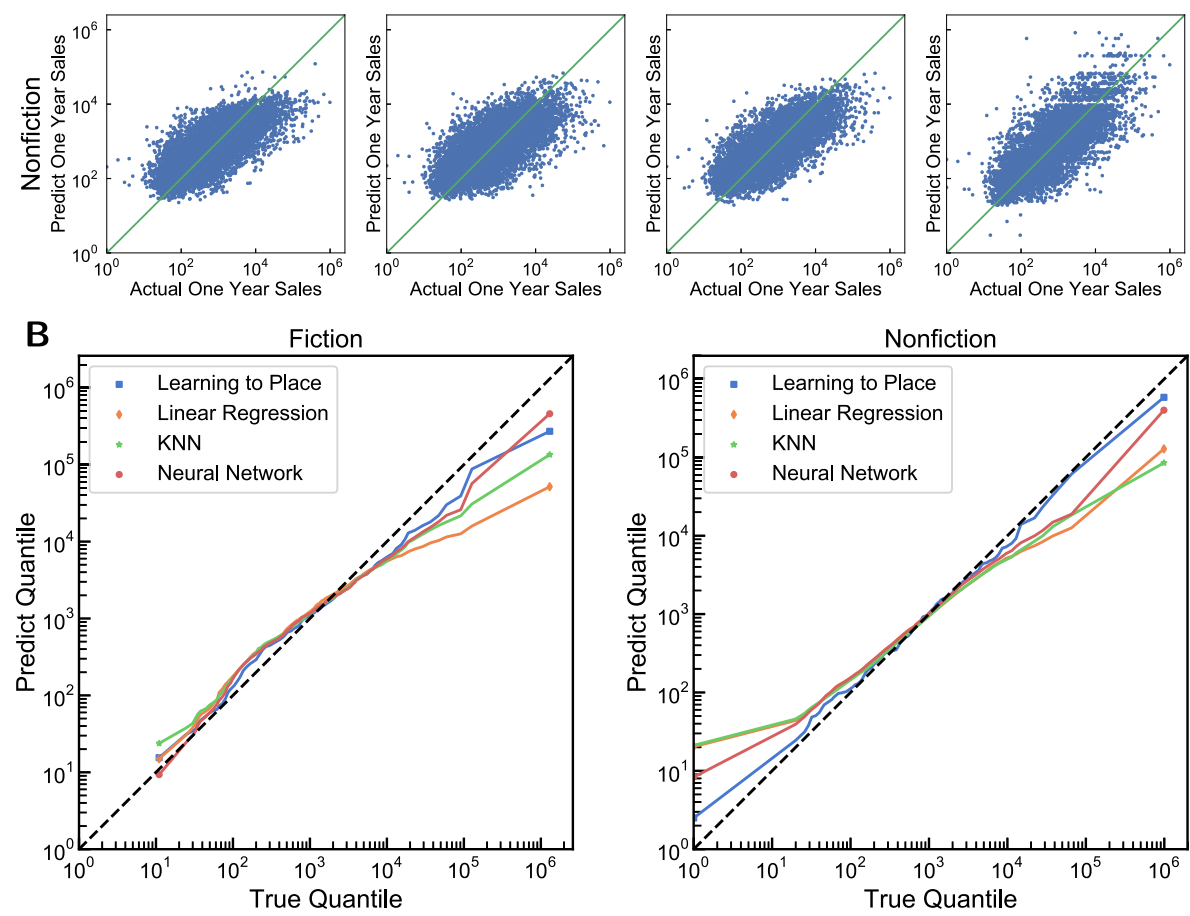

Figure 7 Model results of one year sales for fiction and nonfiction books. We compare Learning to Place against three baseline methods (Linear Regression, KNN, Neural Network). (A) Actual vs prediction scatter plots. All three baseline methods systematically underpredict at high-end to a certain extent while this underprediction is absent in Learning to Place. (B) Quantile-Quantile plot. For fiction, on the high-end sales, neural network and Learning to Place are the closest to the ground truth (45 degree line). For nonfiction, Learning to Place is the closest to the ground truth. KNN and Linear Regression at the high-end systematically have lower predictive power for both fiction and nonfiction

Feature importance for different genres: We also apply Learning to Place on selected genres and look at the feature importance difference between different genres. We select the five largest genres under fiction (Mystery, Thriller, Fantasy, Historical, Literacy) and nonfiction (Biography, Business, Cooking, History, Religion) respectively and obtain the feature importance for each genre.

Figure $9 \mathrm{~B}$ and $\mathrm{C}$ shows normalized accuracy score using each feature group for each genre. We find that across all genres, Imprint is the most important feature group, followed by previous sales and visibility; with all other feature groups having limited importance. We do observe, however, small but insightful differences between genres. Within fiction, we see that for Fantasy and Thrillers, author's visibility is much more important than with Literary genre. Thrillers and Mystery \& Detective have higher importance in previous sales than in other genres, possibly due to the fact that serial books are common in these two genres. For nonfiction genres, we see that for all genres Imprint is the most important 

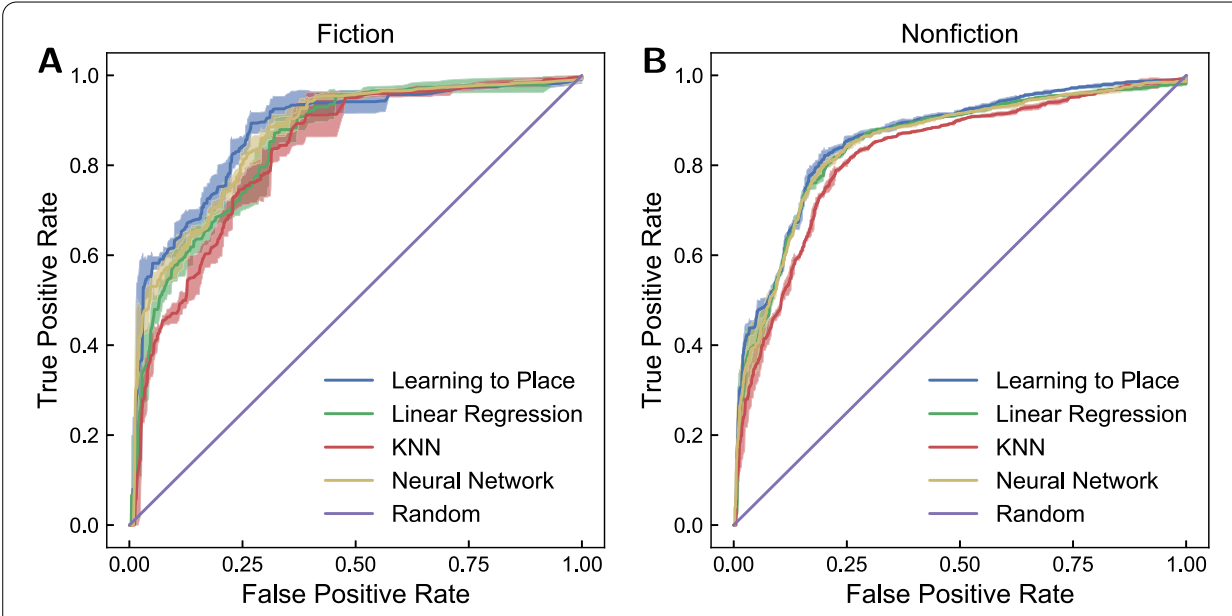

C

\begin{tabular}{lllll}
\hline Category & \multicolumn{3}{c}{ Fiction } & \multicolumn{3}{l}{ Nonfiction } \\
\cline { 2 - 5 } Method & AUC score & High-end RMSE & AUC score & High-end RMSE \\
\hline KNN & $0.83 \pm 0.03$ & $0.71 \pm 0.06$ & $0.82 \pm 0.01$ & $0.62 \pm 0.02$ \\
Linear Regression & $0.85 \pm 0.01$ & $0.60 \pm 0.07$ & $0.85 \pm 0.01$ & $0.52 \pm 0.03$ \\
Neural Network & $0.86 \pm 0.03$ & $0.50 \pm 0.12$ & $0.85 \pm 0.01$ & $0.48 \pm 0.05$ \\
Learning to Place & $\mathbf{0 . 8 8} \pm \mathbf{0 . 0 1}$ & $\mathbf{0 . 4 6} \pm \mathbf{0 . 1 3}$ & $\mathbf{0 . 8 6} \pm \mathbf{0 . 0 1}$ & $\mathbf{0 . 4 3} \pm \mathbf{0 . 0 1}$ \\
\hline
\end{tabular}

Figure 8 ROC curve and measurement table for one year sales. ROC curve for (A) fiction books and (B) nonfiction books. The Learning to Place method performs better than Linear Regression as well as the KNN Baseline. Neural Network achieves comparable ROC curves. Band around the curve represents the standard deviation of the score across 5-fold cross validation. (C) Measurement Table comparing the performance of KNN, Linear Regression, Neural Network and Learning to Place. A higher AUC is better; a lower RMSE is better. We see that Learning to Place outperforms in every measure

feature group. Biography relies more on the author's visibility than his/her previous sales; while Religion shows the exact opposite pattern: previous sales matters more than author visibility.

Since we have features in three main categories: author, book and publisher, we can also look at the importance for each of these categories. To achieve this, we train three models, each including only one feature category. We then predict the sales of each book using each of these three models separately, and obtain the absolute error $E_{\text {author }}, E_{\mathrm{book}}, E_{\text {publisher }}$ compared to the true sales of the book, and normalize these three errors so that they sum up to one. Finally, we use a ternary plot to inspect the source of errors for different books.

Figure 10 shows the ternery plot for books in different genres in fiction and nonfiction. To help interpret the plot, we color the books based on their actual sales. We observe that for all genres, the top corner has the highest density, meaning that if we rely only on the book feature category, we have the largest prediction error, showing that imprint and author feature are very important sales predictors. The left corner has the second highest density for most genres, meaning that for many books, having only publisher information is not sufficient to obtain a good prediction. The middle of the triangle has the third highest density, which is seen more clearly in nonfiction genres. Most books in the middle area are high selling books, which indicates that true excellence in book sales require excelling in all three dimensions: author, book and publisher. 


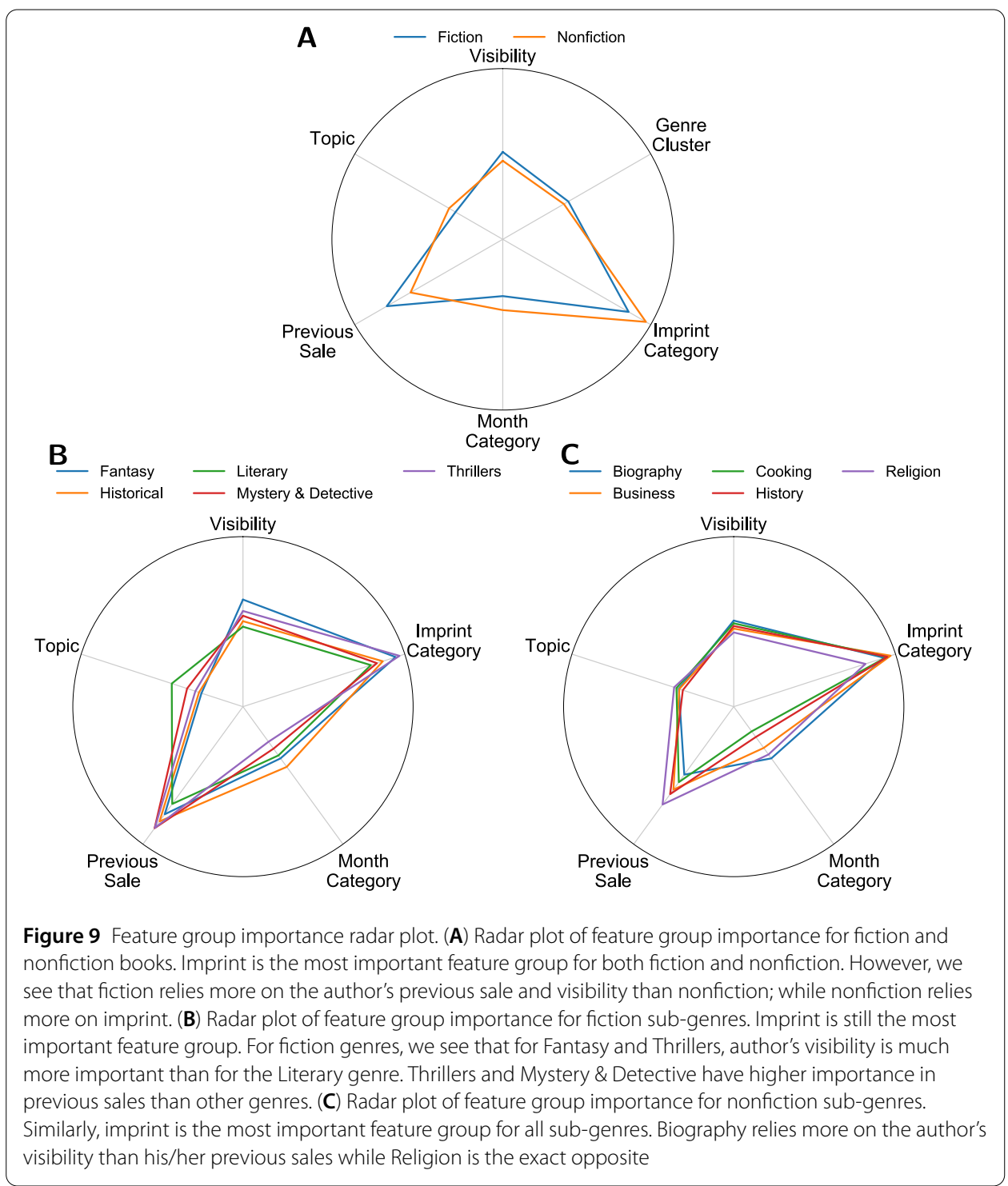

\subsection{Case studies}

Next we illustrate the predictive power of our algorithm on specific books. This exercise helps us understand the algorithm, together with its limitations, offering an intuition of how different features contribute to the success of a particular book (Fig. 11).

We calculate the modified $\mathrm{z}$-score of $\log$ error $(\log$ (predict) $-\log ($ true $)$ ) for each book to investigate individual prediction performance. Selecting z-score $=2$ as the threshold, we find that only $13.7 \%$ of the fiction books and $14.6 \%$ of the nonfiction books have $\mathrm{z}$-score greater than 2, documenting the overall good performance for our predictions (see also Fig. 11 where we color books with a z-score $>2$ dark green in the top-left scatter plot for both fiction and nonfiction books).

For both fiction and nonfiction, a book is likely to have higher sales if all of its features are in the high range. Consider the fiction book Precious Gifts by Danielle Steel, for which we predict sales of around 96,000, very close to the real sales of around 110,000. Danielle Steel is a New York Times bestselling author of multiple books, with high visibility (more than $10^{6}$ cumulative Wikipedia pageviews) and outstanding sales history record (more than $10^{6}$ 


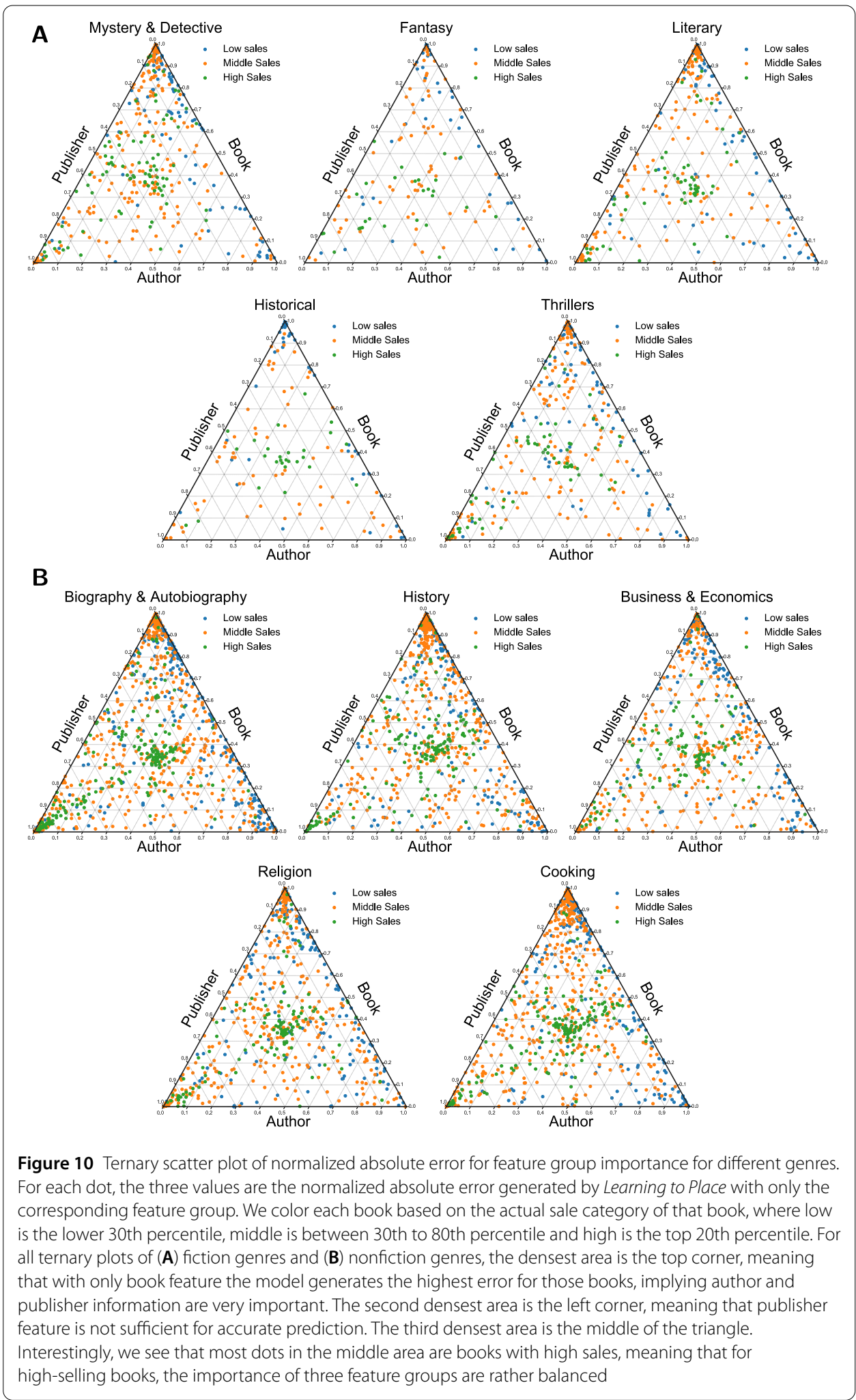

copies sold). The publisher of the book Delacorte Press is in the top 10th percentile of the median sales of books published and top 25th percentile in the number of books published (Fig. 4), and the genre romance is one of the highest selling genres. Though a December 


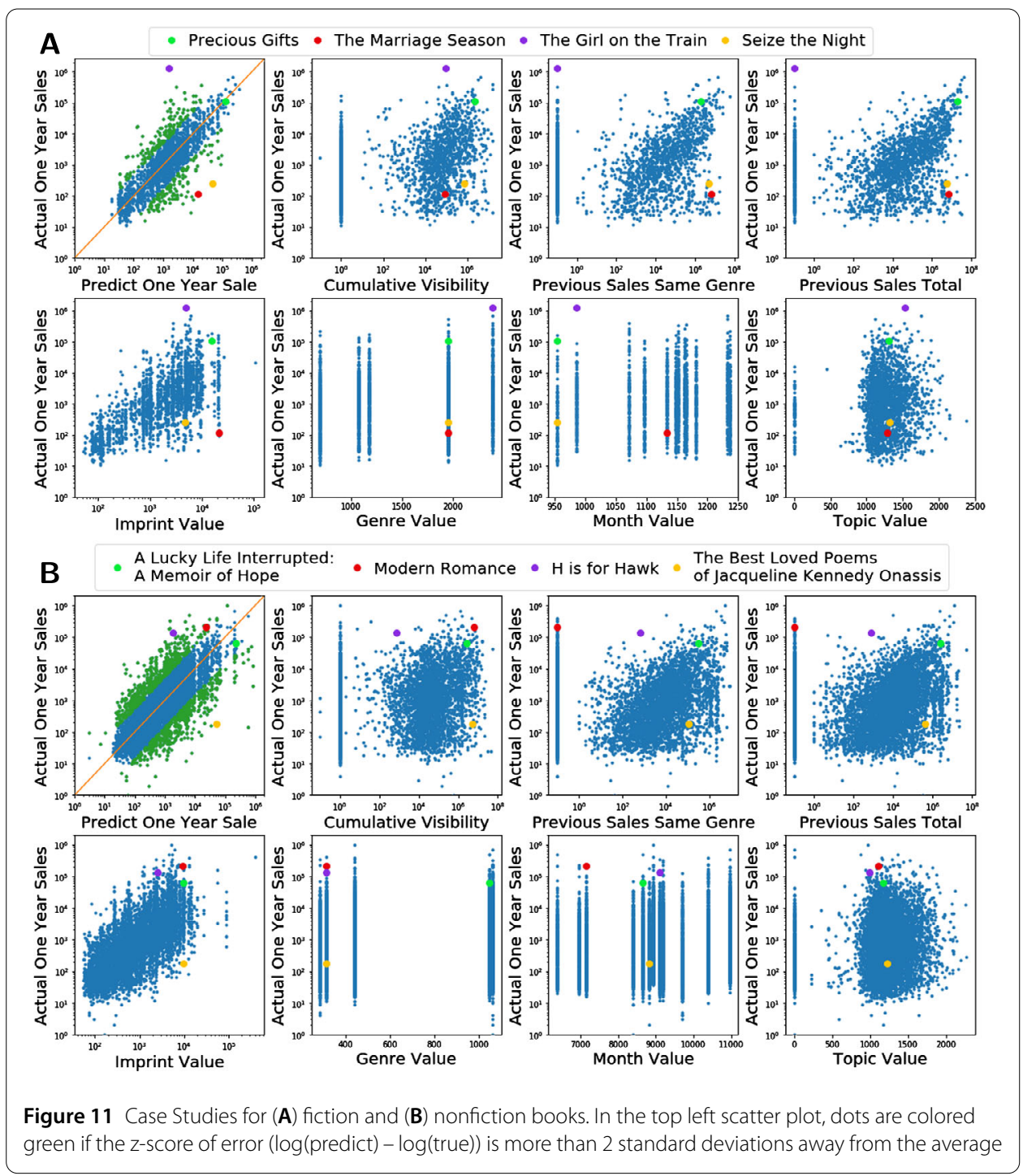

publishing date does not favor the highest sales, all other features push this book towards a high selling status.

For an accurate prediction in nonfiction, consider the biography A Lucky Life Interrupted: A Memoir of Hope by Tom Brokaw published by Random House. The author has high visibility (more than $10^{6}$ Wikipedia pageviews) and high previous sales (more than $10^{5}$ copies sold); additionally the publisher Random House is in the top 10th percentile in both the number and median sales of books published; and biography is one of the most selling genres in nonfiction. So it is no surprise that it was a New York Times bestseller. Our model predicts for this book sales around 66,000, fairly close to the true sales of 63,000.

However, excellence in each criteria does not always guarantee a high-selling book. Consider the fiction The Marriage Season by Linda Lael Miller. She has more than $10^{4}$ Wikipedia pageviews and more than $10^{6}$ copies sold previously. The publisher of the book Harlequin Books is in the top 10th percentile in terms of the median sales of books published and above median in terms of the number of books published, indicating it is an experienced imprint with good reputation. The genre of the book belongs to the second 
high-selling genre cluster. Our model predicts it will sell 14,000 copies; however, it sold only about 110 copies in one year. According to the top review on Amazon of this book, one reader remarked that it was "boring when it comes to romance and the depth of characters". In other words, the quality of the book failed to appeal to the readers, something our features fail to capture.

We also find that visibility can be very important. Consider, for example, Modern Romance by Aziz Ansari, an author without previous sales, but a well-known actor and comedian, with more than $10^{6}$ Wikipedia pageviews. Together with the fact that the publisher is Penguin Press, which is one of the highest ranked publishers in Nonfiction (top 10th percentile in both median sales and number of books published), this book ended up being a bestseller. Our model underpredicts its sales (prediction is around 23,000 while the true sales is around 211,000), because most bestselling books are by authors with high previous sales.

Finally, there are always surprising cases in publishing industry, for example, the phenomenal bestseller The Girl on the Train by Paula Hawkins. She worked as a journalist for the Times in the Business section, having some visibility (about $10^{4}$ pageviews) before her book. She has written several romantic comedy fiction books but under the pseudonym Amy Silver. Therefore, when we look at the features of The Girl on the Train, we find that she has no previous sales in Thrillers \& Suspense. The book was out in January-a month that is very unlikely to lead to high one-year sales. Yet the publisher of the book, Riverhead Books, has a good reputation (top 25th percentile in both median sales and number of books published). However, since there are not many fiction authors that obtain such tremendous sales without any publishing history, our model greatly underpredicts her book (prediction around 1500 vs. actual sales 1,300,000). In nonfiction, we have $H$ is for Hawk by Helen Macdonald published by Grove Press, in which case both the visibility and previous sales of the author are not very high (about $10^{2}$ pageviews and copies sold previously); the publisher is strong but not among the top publishers (top 25th percentile in median sales and above median in number of books published); the genre and topic of the book is not the most-selling one. However, this book became a bestseller while our model fails to predict it (our prediction is around 3000 and actual sales was 140,000).

Additionally, note that some of the incorrect predictions are rooted in data error. Consider for example for Seize the Night by Sherrilyn Kenyon, an author with high visibility (more than $10^{5}$ pageviews) and previous sales (more than $10^{6}$ copies sold), good imprint (St. Martins Press is in the top 10th percentile in number of books and top 25th percentile in median sales of books published), so our model predicts sales of 45,000 while the book only sold about 200 copies. With further inspection, we found out that the novel is originally published in 2004, not 2015 as Bookscan recorded, and has sold well. Similarly, nonfiction The Best Loved Poems of Jacqueline Kennedy Onassis by Caroline Kennedy under Grand Central Publishing, with claimed publication year 2015 in Bookscan is underpredicted: prediction around 53,000 while the database shows the actual sales around 180 . It turns out that the book was originally published in 2001 and was a New York Times bestseller.

\section{Robustness analysis}

We conducted a robustness experiment to evaluate the model's performance across time. For this, we split the book's publication date in four quarters: (1) January to March, (2) 
Table 1 Robustness experiment. To analyze the robustness of our model assuming a realistic setting, we trained our model using earlier instances and tested it on books published later. We report AUC (top) and High-end RMSE (bottom) scores for models train on qth quarter of the year 2015 and test on the $q+1$ th quarter. We observe that Learning to Place almost always outperform other methods

\begin{tabular}{lllllll}
\hline Quarter & Quarter 2 & Quarter 3 & Quarter 4 & Quarter 2 & Quarter 3 & Quarter 4 \\
\hline Category & Fiction (AUC) & & \multicolumn{4}{l}{ Nonfiction (AUC) } \\
KNN & 0.83 & 0.82 & 0.82 & 0.81 & 0.80 & 0.82 \\
Linear Regression & 0.85 & 0.83 & 0.86 & $\mathbf{0 . 8 5}$ & $\mathbf{0 . 8 4}$ & $\mathbf{0 . 8 6}$ \\
Neural Network & $\mathbf{0 . 8 8}$ & 0.83 & 0.73 & 0.83 & 0.83 & 0.85 \\
Learning to Place & $\mathbf{0 . 8 8}$ & $\mathbf{0 . 8 5}$ & $\mathbf{0 . 8 8}$ & $\mathbf{0 . 8 5}$ & 0.83 & 0.85 \\
Category & Fiction (High-end RMSE) & & Nonfiction (High-end RMSE) & \\
KNN & 0.60 & 0.91 & 1.03 & 0.71 & 0.72 & 0.77 \\
Linear Regression & 0.61 & 0.77 & 0.89 & 0.58 & 0.61 & 0.58 \\
Neural Network & 0.44 & 0.81 & 2.83 & 0.71 & 0.57 & 0.63 \\
Learning to Place & $\mathbf{0 . 4 2}$ & $\mathbf{0 . 7 1}$ & $\mathbf{0 . 4 5}$ & $\mathbf{0 . 4 9}$ & $\mathbf{0 . 5 1}$ & $\mathbf{0 . 6 2}$ \\
\hline
\end{tabular}

April to June, (3) July to September and (4) October to December. We conduct experiments training on quarter $q$ and testing on quarter $q+1$ (e.g., training on books published in January to March and testing on books published in April to June.)

We also measured the feature importance for all four quarters to observe their stability. Since after splitting the number of data points under individual genre available for the measurement is smaller, here we focus on the feature importance for fiction and nonfiction.

The model performance for different quarters is summarized in Table 1. We find that the results are stable across different quarters and Learning to Place almost always outperforms other methods. The feature importance of fiction and nonfiction for different quarters are in Additional file 1, Fig. 4, demonstrating that the feature importance is generally stable across time.

\section{Conclusions}

In this paper, our goal was to develop tools capable of predicting a book's sales prior to the book's publication, helping us understand what factors contribute to the success of a book. To do that, we first extracted the pertinent features of each book, focusing on those that are available to readers before or at publication, and employed a new machinelearning approach, Learning to Place, which solves the prediction problem of heavy-tailed outcome distributions [30].

We extracted features from three categories: author, book and publisher. For the author feature group, we measure the visibility and the previous sales of an author; for the book feature group, we consider the genre, topic and publication month of the book; and for the publisher, we measure the reputation of the publisher.

An important challenge of our prediction task is that we have far more low-selling books than high-selling books; therefore, traditional methods like Linear Regression systematically underpredict high-selling books. We employed the Learning to Place algorithm to correct this limitation. For this, we first obtain the pairwise preferences between books, and use it to assign the place of the book compared to other books and obtain its sales prediction. Similar pairwise relations has been used to rank items using tournament graphs [34], inferring fitness of each instance [35], and optimizing constraints of pairwise relations [36]. However, our task aims to accurately estimate book sales. We found that with our Learning to Place algorithm, we can predict the sales of fiction and nonfiction 
fairly accurately and the algorithm does not suffer from systematic underprediction for high-selling books comparing to Linear Regression and $k$-nearest neighbors.

The developed framework also allows us to understand the features driving the book sales. We found that for both fiction and nonfiction, the publisher quality and experience is the most important feature, due to the fact that the publisher both pre-selects and advertises the book. Previous publishing history and visibility of the author are very important as well since readers are more likely to read books written by experienced authors or celebrities. The genre, topic and publication month of the book, however, have only limited influence on the sales of the book.

We also found that the feature importance are slightly different for different genres. For Thrillers and Mystery \& Detective, author's visibility and previous sales are more important than in other fiction genres. In nonfiction genres, Biography relies more on visibility than previous sales; while this is the opposite for History. Using the ternery plot we also find that author and publisher are very important for most books and for most of high selling books, author, publisher and book contributes equally to the sales.

We expect our methodology and findings to serve as a starting point towards a better understanding of the mechanisms driving the publishing industry and reader preferences. We hope that our research will inspire more investigation in the success of books and authors, helping us to create a more innovative, predictive as well as profitable environment for authors as well as for the publishing industry.

\section{Supplementary information}

Supplementary information accompanies this paper at https://doi.org/10.1140/epjds/s13688-019-0208-6.

Additional file 1. Supplementary information (PDF 987 kB)

\section{Acknowledgements}

We wish to thank Douglas Abrams, Akos Erdös and Adam Halmes for offering insider's view to the publishing word and helping us understand it better, Alice Grishchenko for helpful visualizations, Peter Rupert and other colleagues at the CCNR, especially those in the success group, for valuable discussions and comments.

\section{Funding}

This research was supported by Air Force Office of Scientific Research (AFOSR) under agreements FA9550-15-1-0077 and FA9550-19-1-0359, John Templeton Foundation under agreement 61066 and DARPA under agreement N66001-16-1-4067. ALB has received funding from the European Union's Horizon 2020 research and innovation programme under grant agreement No 810115-DYNASNET.

\section{Competing interests}

ALB is the founder and holds shares of three startups that use network science and data science tools. The other authors declare that they have no competing interests.

\section{Authors' contributions}

All authors designed the research. XW and BY, prepared and cleaned the data. XW, BY, OV and TE-R designed the algorithm, conducted the experiments and prepared the figures. XW and A-LB wrote the manuscript. All authors read and approved the final manuscript.

\section{Author details}

${ }^{1}$ Center for Complex Network Research and Department of Physics, Northeastern University, Boston, USA. ${ }^{2}$ College of Computer and Information Science, Northeastern University, Boston, USA. ${ }^{3}$ Department of Medicine, Brigham and Women's Hospital, Harvard Medical School, Boston, USA. ${ }^{4}$ Center for Network Science, Central European University, Budapest, Hungary.

\section{Publisher's Note}

Springer Nature remains neutral with regard to jurisdictional claims in published maps and institutional affiliations. 


\section{References}

1. Statista: U.S. Book Industry/Market—Statistics \& Facts. https://www.statista.com/topics/1177/book-market/ [Online; accessed 23-May-2018] (2018)

2. Ashok VG, Feng S, Choi Y (2013) Success with style: using writing style to predict the success of novels. In: Proceedings of the 2013 conference on empirical methods in natural language processing, pp 1753-1764

3. Clement M, Proppe D, Rott A (2007) Do critics make bestsellers? Opinion leaders and the success of books. J Media Econ 20(2):77-105

4. Chevalier JA, Mayzlin D (2006) The effect of word of mouth on sales: online book reviews. J Mark Res 43(3):345-354

5. Kovács B, Sharkey AJ (2014) The paradox of publicity: how awards can negatively affect the evaluation of quality. Adm Sci Q 59(1):1-33

6. Shehu E, Prostka T, Schmidt-Stölting C, Clement M, Blömeke E (2014) The influence of book advertising on sales in the German fiction book market. J Cult Econ 38(2):109-130

7. Nakamura L (2013) "Words with friends": socially networked reading on Goodreads. PMLA 128(1):238-243

8. Beck J (2007) The sales effect of word of mouth: a model for creative goods and estimates for novels. J Cult Econ 31(1):5-23

9. Schmidt-Stölting C, Blömeke E, Clement M (2011) Success drivers of fiction books: an empirical analysis of hardcover and paperback editions in Germany. J Media Econ 24(1):24-47. https://doi.org/10.1080/08997764.2011.549428

10. Dellarocas C, Zhang XM, Awad NF (2007) Exploring the value of online product reviews in forecasting sales: the case of motion pictures. J Interact Mark 21(4):23-45. https://doi.org/10.1002/dir.20087

11. Abel F, Diaz-Aviles E, Henze N, Krause D, Siehndel P (2010) Analyzing the blogosphere for predicting the success of music and movie products. In: Advances in social networks analysis and mining (ASONAM), 2010 international conference on. IEEE Press, New York, pp 276-280

12. Park J, Ciampaglia GL, Ferrara E (2016) Style in the age of instagram: predicting success within the fashion industry using social media. In: Proceedings of the 19th ACM conference on computer-supported cooperative work \& social computing. CSCW'16. ACM, New York, pp 64-73. https://doi.org/10.1145/2818048.2820065

13. Fourt LA, Woodlock JW (1960) Early prediction of market success for new grocery products. J Mark 25(2):31-38

14. Mestyán M, Yasseri T, Kertész J (2013) Early prediction of movie box office success based on Wikipedia activity big data. PLOS ONE 8(8):71226

15. Varol O, Ferrara E, Menczer F, Flammini A (2017) Early detection of promoted campaigns on social media. EPJ Data Sc 6(1):13

16. Yucesoy B, Wang X, Huang J, Barabási A-L (2018) Success in books: a big data approach to bestsellers. EPJ Data Sci $7(1): 7$

17. Group, B.I.S.: Complete BISAC Subject Headings List, 2017 Edition. http://bisg.org/page/BISACEdition [Online; accessed 4-October-2017] (2017)

18. Wikipedia: Data dumps. https://meta.wikimedia.org/wiki/Data dumps [Online; accessed 13-April-2018] (2018)

19. Wikipedia: API:Main page. https://www.mediawiki.org/wiki/API:Main_page [Online; accessed 13-April-2018] (2018)

20. Spoerri A (2007) What is popular on Wikipedia and why? First Monday 12(4)

21. Keegan B, Gergle D, Contractor N (2013) Hot off the Wiki: structures and dynamics of Wikipedia's coverage of breaking news events. Am Behav Sci 57(5):595-622

22. Yucesoy B, Barabási A-L (2016) Untangling performance from success. EPJ Data Sci 5(1):17

23. Bird S, Klein E, Loper E (2009) Natural language processing with Python, 1st edn. O'Reilly Media, Sebastopol

24. Manning CD, Schütze H (1999) Foundations of statistical natural language processing. MIT Press, Cambridge

25. Lloyd S (1982) Least squares quantization in pcm. IEEE Trans Inf Theory 28(2):129-137

26. King G, Zeng L (2001) Logistic regression in rare events data. Polit Anal 9(2):137-163

27. Hsu D, Sabato S (2016) Loss minimization and parameter estimation with heavy tails. J Mach Learn Res 17(1):543-582

28. Maalouf M, Homouz D, Trafalis TB (2018) Logistic regression in large rare events and imbalanced data: a performance comparison of prior correction and weighting methods. Comput Intell 34(1):161-174

29. Schubach M, Re M, Robinson PN, Valentini G (2017) Imbalance-aware machine learning for predicting rare and common disease-associated non-coding variants. Sci Rep 7(1):2959

30. Wang X, Varol O, Eliassi-Rad T (2019) L2P: an algorithm for estimating heavy-tailed outcomes. arXiv preprint. arXiv:1908.04628

31. Breiman L (2001) Random forests. Mach Learn 45(1):5-32

32. Mosteller F, Tukey JW (1968) Data analysis, including statistics. Handb Soc Psychol 2:80-203

33. Stone M (1974) Cross-validatory choice and assessment of statistical predictions. J R Stat Soc, Ser B, Methodol 36:111-147

34. Cohen WW, Schapire RE, Singer Y (1998) Learning to order things. In: Advances in neural information processing systems, pp 451-457

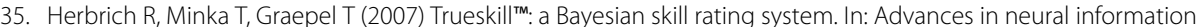
processing systems, pp 569-576

36. Joachims T (2002) Optimizing search engines using clickthrough data. In: Proc of the 8th ACM SIGKDD intl conf on knowledge discovery and data mining. ACM, New York, pp 133-142 